\title{
Review Article \\ Fuzzy-Logic-Based Control, Filtering, and Fault Detection for Networked Systems: A Survey
}

\author{
Yuqiang Luo, ${ }^{1}$ Zidong Wang, ${ }^{2,3}$ Guoliang Wei, ${ }^{1}$ Bo Shen, ${ }^{4}$ Xiao He, \\ Hongli Dong, ${ }^{6,7}$ and Jun $\mathrm{Hu}^{7,8}$ \\ ${ }^{1}$ Shanghai Key Lab of Modern Optical System, Department of Control Science and Engineering, \\ University of Shanghai for Science and Technology, Shanghai 200093, China \\ ${ }^{2}$ Department of Computer Science, Brunel University London, Uxbridge, Middlesex UB8 3PH, UK \\ ${ }^{3}$ Communication Systems and Networks (CSN) Research Group, Faculty of Engineering, King Abdulaziz University, \\ Jeddah 21589, Saudi Arabia \\ ${ }^{4}$ School of Information Science and Technology, Donghua University, Shanghai 200051, China \\ ${ }^{5}$ Department of Automation, Tsinghua University, Beijing 100084, China \\ ${ }^{6}$ College of Electrical and Information Engineering, Northeast Petroleum University, Daqing 163318, China \\ ${ }^{7}$ Research Institute of Intelligent Control and Systems, Harbin Institute of Technology, Harbin 150001, China \\ ${ }^{8}$ Department of Applied Mathematics, Harbin University of Science and Technology, Harbin 150080, China
}

Correspondence should be addressed to Guoliang Wei; guoliang.wei1973@gmail.com

Received 29 January 2015; Accepted 28 April 2015

Academic Editor: Anna Pandolfi

Copyright (C) 2015 Yuqiang Luo et al. This is an open access article distributed under the Creative Commons Attribution License, which permits unrestricted use, distribution, and reproduction in any medium, provided the original work is properly cited.

This paper is concerned with the overview of the recent progress in fuzzy-logic-based filtering, control, and fault detection problems. First, the network technologies are introduced, the networked control systems are categorized from the aspects of fieldbuses and industrial Ethernets, the necessity of utilizing the fuzzy logic is justified, and the network-induced phenomena are discussed. Then, the fuzzy logic control strategies are reviewed in great detail. Special attention is given to the thorough examination on the latest results for fuzzy PID control, fuzzy adaptive control, and fuzzy tracking control problems. Furthermore, recent advances on the fuzzy-logic-based filtering and fault detection problems are reviewed. Finally, conclusions are given and some possible future research directions are pointed out, for example, topics on two-dimensional networked systems, wireless networked control systems, Quality-of-Service (QoS) of networked systems, and fuzzy access control in open networked systems.

\section{Introduction}

Networked Control Systems. In recent years, with the rapid development of the network technology and application, networked systems have received comprehensive attention from researchers and engineers, and plenty of results have been reported; see, for example, [1-3]. The networked control system (NCS) is an integration of control science, network technology, and computer science. A NSC features many advantages such as simple installation and maintenance, reduced weight and power requirements, reduction of installation and maintenance costs, and decentralization of function $[4,5]$. In a typical NCS, sensors, plants, controllers, and actuators are connected through communication networks undertaking the main transmission task between system elements. Broadly speaking, when the control loop is closed via a communication channel, the controlled system can be labeled as a NCS [6]. Due to its practicability and validity, the NCS has found various practical applications including industrial automation, remote surgery, building automation, mobile sensor networks, robot, intelligent vehicle systems, advanced aircraft, and so forth [7-9]. However, the presence of the network also introduces some disadvantages, for instance, time-varying network-induced delays, quantization, aperiodic sampling, packet dropouts, and channel fading. These network-induced constraints might seriously deteriorate the performance of the systems and sometimes even lead to instability. Therefore, analysis and design of 
NCSs have gained increasing concern in the past years; see, for example, [10].

Roughly speaking, the NCSs can be classified into the following two types according to the existing infrastructure in practice, namely, fieldbus control systems (FCSs) and Industrial Ethernet (IE). A fieldbus-based NCS is a special system whose control loops are closed through a fieldbus. Due to their generality and efficiency, FCSs have been widely used in automation. Industrial control practice has demonstrated that the manufacturing and production have benefited a lot from FCSs in the past decades. With the advent of fieldbus technologies, the traditional control structure has been changed remarkably. By employing fieldbus technologies, the field equipment, field instruments, and measurement device can be linked into a local network system so as to render the real-world applications of NCSs possible. There are many kinds of FCSs; for example, an overview of the current state of these fieldbus networks has been presented in [11], where various advantages of FCSs over point-to-point connections have been given. For details of the FCSs, readers are referred to $[12,13]$.

The Industrial Ethernet is another important type of NCSs, which also plays an important role in the actual production and scientific research. Birth and development of IE provide practical basis for NCSs and back up the applications of remote monitoring and control, networked manufacturing, intelligent control, and so forth. Compared with the traditional networks, IE network equipping modern intelligent switching technologies owns a variety of advantages such as low cost, easy extensibility, and flexible and fast networking. On the other hand, the widespread use of the existing industry standards has significantly reduced the compatibility risks and network complexity of IE networks by replacing some proprietary standards with the common standards. Because of their real-time performance, high reliability, and openness, IE networks have been widely applied in industrial control and gained significant achievements; see, for example, $[14,15]$.

Fuzzy Control Systems. Since the introduction of fuzzy set theory by Zadeh [16-18], the fuzzy logic theory has been developed in a variety of directions. Now, the fuzzy logic theory has found widespread applications in the control engineering, signal and information processing, pattern recognition, expert system, decision making, and so on. As one of the most successful applications of fuzzy sets and fuzzy logic, the research on fuzzy control problems has undergone great progress in the past decades [19]. Since the fuzzy-logicbased control was first used in the controller design for the steam engine in 1974 [20,21], it has been extensively applied to system modeling, intelligence control, system identification, pattern recognition and classification, neural network, and so forth. Compared with conventional crisp control, fuzzylogic-based control can model humans' experience more accurately in a linguistic manner, thereby providing an efficient way to realize the intelligent control in industrial application.
In general, the fuzzy control system can be classified into two types: the model-free and the model-based fuzzy control system. The model-free fuzzy control scheme has witnessed great developments in recent years because, under certain circumstances, it outperforms other conventional model-free approaches such as the nonlinear adaptive or PID control. A large number of important results have appeared on this issue; see, for example, [22, 23]. The model-based fuzzy control systems include three types: type-1, type-2, and type-3 [24]. Presentation of these three different types of fuzzy control systems can be found in [25], from which one can know that type- 2 fuzzy control system is actually a special case of type- 1 . There are many applications of type- 1 fuzzy control systems; see, for example, [26-29]. The type-2 fuzzy control system has been widely applied in practice; see, for example, [30-34]. In fact, it was first proposed by Sugeno in [25] and first used for automobile tracking control [35]. The type-3 fuzzy control system is actually the T-S fuzzy model, which has proven to be an extremely effective method for tackling nonlinear NCSs. As pointed out in [36], the Takagi-Sugeno (T-S) model is able to approximate any smooth nonlinear function to any degree of accuracy in any convex compact region. By using a set of local linear models which are smoothly connected by nonlinear fuzzy membership functions to present a nonlinear plant, the T-S fuzzy model has brought the analysis and synthesis of nonlinear systems into a unified framework. Through decades of developments, the T-S fuzzy model has enjoyed an extensive utilization; see, for example, [37-40] for some original literature and $[41,42]$ for the latest literature.

Organization of This Paper. The main objective of this paper is to provide a general overview on the advance of fuzzy-logicbased methodology in networked systems. A brief description of characteristics and advantages of the networked systems is given, where two types of practically used networked systems are introduced. Some latest literature relating to network-induced phenomena of NCSs has been presented. Advances of fuzzy-logic-based control, filtering, and fault detection for networked systems are summarized, and the recent progresses of applications and improvements of the fuzzy-logic-based control in the NCSs have been reviewed.

The remainder of this paper is organized as follows. In Section 2, network-induced phenomena such as packet dropouts, time delays, signal quantization, and link failures are discussed. Section 3 introduces latest results on fuzzylogic-based control for NCSs with emphasis on three common control schemes, namely, PID control, adaptive control, and tracking control. In Section 4, the fuzzy-logic-based filtering and fault detection problems are examined and the corresponding results are presented. In Section 5, some concluding remarks of this paper are drawn and several possible directions for further research are pointed out.

\section{Network-Induced Phenomena}

Network-induced phenomena emerge for mainly two reasons. For one thing, because the network itself is a nonlinear dynamic system, its Quality-of-Service depends on many 
conditions such as cable quality, bandwidth capacity, and link quality. Therefore, any variation (e.g., cable aging, interface failures, limited bandwidth, and network congestion) would affect the application service of networked systems. For another thing, the risk and frequency of the occurrence of faults/failures in networked systems are higher than those for traditional systems due to the inherent peculiarity of the NCSs such as multiple components, large scale, wide distribution, and high complexity. Moreover, the data in the open condition of network transferring is vulnerable to malicious attacks and theft. Therefore, it is very necessary to address the data security of NCS. For these reasons, the network-induced constraints of NCSs have acquired unprecedented interests in the recent years. In this section, the network-induced phenomena will be discussed, which include packet dropouts, time delays, signal quantizations, actuator/sensor/link failures channel fading, and data security.

As an important network-induced constraint, the packet dropout is an inevitable problem in NCSs due to the channel noise and the network congestion. It is generally known that packet dropouts have significant impacts on the analysis and synthesis of NCSs. If it is not properly handled, the packet dropout would degrade the NCS performance and even destabilize systems. On the other hand, the communication time delay is a well-known kind of network-induced phenomena that exist universally in the networked systems. According to the way the delays occur, there are some different types of delays including constant delays, timevarying delays, discrete delays, and distributed delays. In the past few decades, the topics of research on time-delay systems have been attracting constant attention from the control community. Because of the universality and complexity of the time delays, it is very important and challenging to design effective algorithms so as to reduce the impact from delays on the NCSs. Generally speaking, the existing stability criteria for time-delay systems can be classified into two types, that is, the delay-independent conditions and the delay-dependent ones; see, for example, $[43,44]$. So far, various research methodologies have been developed for studying the time delays and some recent results can be found in $[45,46]$.

In networked systems, it is generally known that the signals are usually quantized before communication. Due to either rounding or truncation of quantization, the quantization effect occurs frequently which poses great challenges on the design of controllers/filters for NCSs. Obviously, the control algorithm specifically designed for a system without quantization will fail to work when the quantization effects do arise. Although the quantization error can be reduced by increasing the number of quantized bits, it may lead to increased power consumption which, in turn, hinder the development of the NCSs [47]. For purpose of reducing energy consumption in NCSs, some power scheduling strategies for quantization have been proposed; see, for example, [47-49]. On the other hand, since the quantization is a critical factor affecting the stability and performance of NCSs, it has become an important issue for researchers to work on control system design with quantization effects; for example, some quantized state feedback control schemes have been employed to solve the stabilization problems in NCSs; see, for example, [50-52].

On another research front of reliable control, the failures in NCS can occur within three main categories, namely, the system devices, sensor nodes, and communication components due primarily to the malfunction such as node crash, low battery power, system reboot, and routing loops. Different from the malfunction or performance failures, the actuator/sensor/link failures often lead to serious and even disastrous outcomes. Therefore, it is of practical significance to look at how to improve the stability, reliability, and intelligence of NCSs with possible failures under different circumstances. In fact, the fault detection, fault diagnosis, and fault tolerant control problems have been widely investigated in the areas especially for NCSs; see, for example, [53-55].

As a common kind of network-induced phenomena, the channel fading has received initial research attention in the context of filter/control designs of NCSs in recent years. Because the wireless communication links are especially susceptible to the fading effects, the channel fading constitutes one of the most dominant features of wireless NCSs. Generally speaking, the channel fading can fall into two major categories, namely, the multipath induced fading and the shadow fading [56]. Since the signal transmitted by a fading channel can be seriously corrupted, design of a reliable controller and filter which is insensitive to fading influence becomes urgent in NCSs. Fortunately, however, some preliminary works have appeared; see, for example, [57, 58].

In the traditional NCSs, the security protection has not been considered seriously, which brings a series of data security problems such as the illegal invasion, information theft, and data tampering in an unencrypted communication condition. If the NCSs are maliciously intruded, the target physical system may be uncontrolled. For these reasons, the data and communication protection of NCSs has stimulated the interests of people in very recent years. Based on the coevolutionary algorithms, the tradeoff problem between the security and the performance of NCSs has been discussed in [59]. With the help of the stochastic game theory, an optimal defense mechanism for the NCSs under jamming attacks has been presented in [60], where the dynamic interactions between the attacker and the defender in NCSs was modeled by a two-player zero-sum stochastic game. In [61], the security decisions has been considered for the identical plantcontroller systems controlled over a shared communication network.

\section{Fuzzy-Logic-Based Control of NCSs}

Compared with traditional control methods, the fuzzy-logicbased control scheme poses the feature of intelligence. In fact, the fuzzy-logic-based control is an important branch of intelligent control, which plays a significant role in enhancing the intelligent level of control systems. In order to obtain better control performance and intelligence, fuzzy-logicbased control has been gradually utilized to improve the traditional control scheme in recent years. In this section, we will provide an overview on the integrations of fuzzy control 


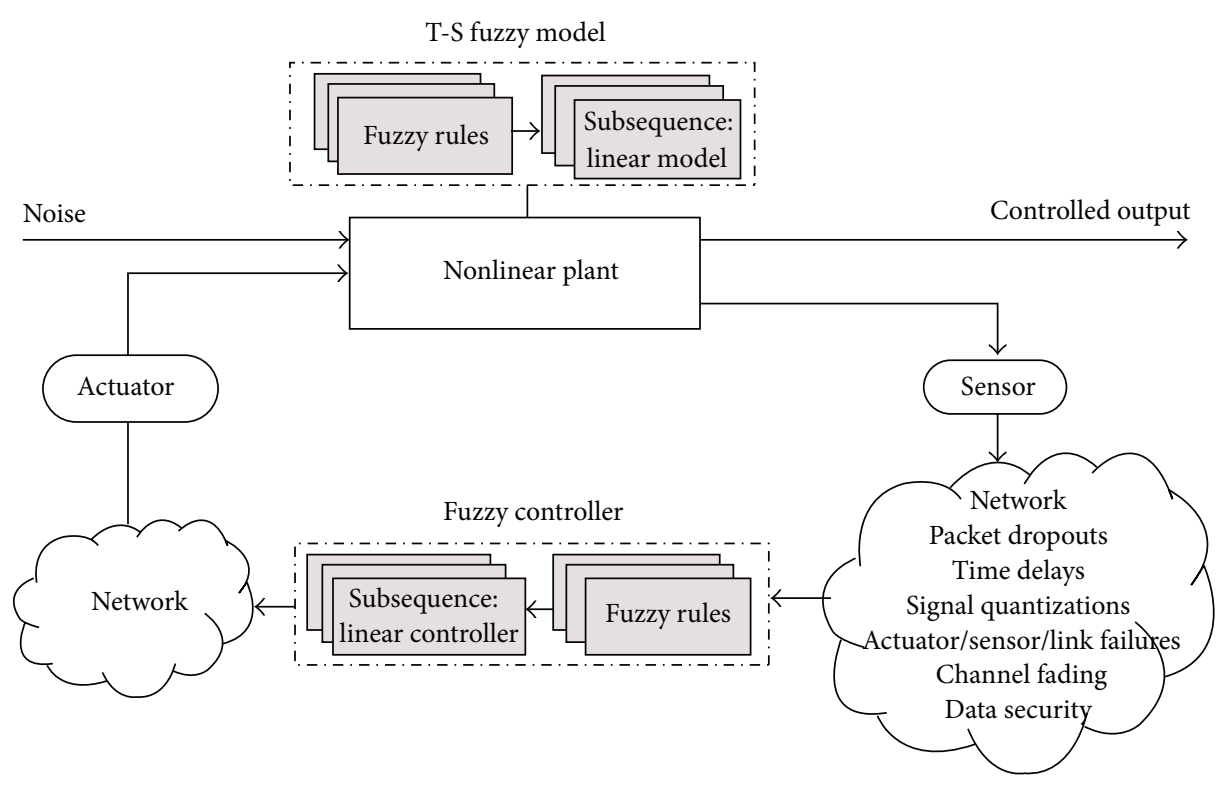

Figure 1: Fuzzy-logic-based control in NCSs.

and some traditional control methods in the NCSs, such as fuzzy PID control, fuzzy adaptive control, and fuzzy tracking control. The general frame of fuzzy-logic-based control in NCSs is shown in Figure 1.

3.1. Fuzzy-Logic-Based General Control. Stability of networked system is a basic yet key issue in NCSs. Compared with the traditional systems, the controller design for NCSs can be influenced by many factors due to the existence of the network-induced phenomena such as uncertain networkload, randomness, and channel constraints. Also, the remote transmission of signals through networks of limited bandwidth brings a great deal of technical difficulties to the NCS control problems. Over the past two decades, researchers have made great efforts on design problems for NCSs and a rich body of works have been reported; see, for example, [6264].

On the other hand, fuzzy-logic-based control has been widely used in NCSs because of its superiority in dealing with knowledge representation and nonlinear approximation. For example, an $H_{\infty}$ output-feedback controller has been designed for a class of nonlinear systems under unreliable communication links in [65], where the communication links between the plant and controller are assumed to be subjected to packet dropouts. A discrete stochastic timedelay nonlinear system with transmitted data dropouts has been stabilized by fuzzy controller in [66]. The probabilistic interval distribution of the communication delay has been taken into account for a class of T-S fuzzy NCSs in [67]. A fuzzy controller has been proposed to stabilize the nonlinear NCSs in [68], where a disturbance attenuation term has also been employed to attenuate the influence of modeling errors and the external disturbances on the system. With the help of the T-S fuzzy approach, a new way to design the quantized controller has been presented for a class of nonlinear systems with quantization errors in [69]. The network-induced delays between sensors and controllers have been modeled by a finite state Markov process in [70], and then a robust mode delay-dependent $H_{\infty}$ controller has been designed for the nonlinear discrete-time NCSs, where the nonlinear systems are modeled by the T-S fuzzy model. An asynchronous output-feedback controller has been designed for NCSs with multiple packet dropouts in [71].

Since the stability analysis and controller synthesis for the networked systems are very important to ensure the convergence of the closed-loop NCSs, so far, a fair amount of research efforts has been devoted to the stability analysis issue of networked systems. Nevertheless, as is well known, quality and performance issues of the NCSs are equally important in practical applications, and also the quantitative evaluation of the control performance is a concern of practical significance. In recent years, many important indices regarding the performance requirements of NCSs have been extensively investigated, such as reliability, disturbance rejection, and guaranteed cost; see, for example, $[10,72-75]$. As a branch of intelligent control, the fuzzy-logic-based control is playing a more and more significant role in improving the performance and intelligence level of NCSs. For instance, a guaranteed cost networked controller has been designed for T-S fuzzy NCSs in [76]. In [77], the robust guaranteed cost control problem has been discussed for a class of nonlinear NCSs to achieve the desired control performance based on the switched T-S fuzzy model. A fuzzy PID controller has been proposed to improve the control performance of NCSs in [78].

3.2. Fuzzy PID Control. PID control is probably the most widely adopted feedback control in today's industrial control systems. To be specific, the PID controller has been successfully employed in a broad range of application domains such as process control, flight control, and instrumentation. 
The main strength of the PID controllers lies in that it is inherently capable of handling practical issues such as actuator saturation and integrator windup [79]. Unfortunately, though the industrial practice has witnessed the effectiveness of PID control for nonlinear systems, it seems inadequate to use PID controller for highly nonlinear systems with precise mathematical models, and there has been a great need to improve the PID control performance. In fact, the new demands from modern control industries have always motivated novel control theories and applications and, with no exception, attempts to improve the PID control techniques have never ceased over the last decades.

With the increasing emergence of nonlinear systems in practical engineering, the need for improving traditional PID controllers is becoming more and more evident. On the other hand, thanks to the approximation ability of the fuzzy systems, nonlinear control laws could be implemented conveniently in a fuzzy control setting. Therefore, the integration of the fuzzy control and the PID control becomes natural to overcome the shortcomings of the traditional PID controller, and such that the PID control will gain more superiority and potential in the NCSs. Some results have recently been reported on this topic. For example, a nonlinear fuzzy PID controller has been designed for systems with nonlinearities and unknown mathematical characteristics in [80]. The gains of the designed PID controller are time-varying by using the fuzzy-logic-based control method, which can greatly improve the transient responses of systems. A nonlinear tuning scheme for PID controllers has been studied based on the fuzzy techniques in [81], and such a scheme has been proved to be indeed superior to some other existing tuning algorithms.

As is well known, it is difficult for the traditional PID controller to adapt to the change of systems due to its constant parameters. However, a fuzzy PID controller can automatically adjust the parameters according to the changes of systems. Some noteworthy works have been reported in the literature. For instance, a new fuzzy PID control strategy has been proposed to improve the control performance of the NCSs in [82]. An optimal fuzzy PID controller has been designed for NCSs in [83], in which the genetic algorithm, particle swarm optimization, and closed-loop performance have been compared. Simulation results have demonstrated that the fuzzy PID controller provides better performance than a simple PID controller, especially in load disturbance suppression. A new class of Smith predictors and a parameter self-tuning fuzzy PID controller have been presented for the NCSs with random time delays in both forward and feedback channels in [84].

3.3. Fuzzy Adaptive Control. As a branch of modern control theory, the adaptive control technique has recently undergone great developments because of its popularity in engineering applications in the fields of digital communication, radar, sonar, earthquake, navigation, industry control, and so on. Generally speaking, there are three methods to accomplish the parameter adaptive control, namely, gain scheduling, model reference control, and self-tuning regulators [85]. Similar to the deterministic robust control, the adaptive control can effectively control the nonlinear systems without exact knowledge of the dynamics [86]. In network environments, since the system parameters are always affected by uncertain dynamics and disturbances, the adaptive control problem for nonlinear NCSs has received more and more attention.

As is well known, the derivation of fuzzy control rules and membership functions is fairly difficult and subjective and is sometimes even painstaking. Therefore, control engineers always expect simple yet efficient methods for fuzzy controller synthesis and knowledge acquisition. Fortunately, fuzzy adaptive control can meet these demands quite well [87] and has therefore attracted increasing attention from researchers. Recently, the idea of fuzzy adaptive control has been progressively applied for the analysis and synthesis of NCSs. For example, an adaptive fuzzy finite-time coordination controller has been designed for the networked teleoperation system with friction and external disturbances in [88], in which a new finite-time synchronization control scheme has been suggested by using the adaptive fuzzy approximation. By adaptively adjusting the scale factor and learning rate of the neuron with fuzzy rulers, an adaptive single neuron PID control algorithm has been modified for the NCSs in [89]. By means of the integration of an online adaptive Smith predictor and a fuzzy controller, a new control scheme has been investigated for NCSs in [90]. Compared with convenient PID controllers, the concerned fuzzy PID controllers possess more capacities to tackle system variations.

3.4. Fuzzy Tracking Control. In recent years, the tracking control has received more and more attention due to its widespread applications in articulated vehicles, mobile robot, hydraulic servo, aerial vehicles and photovoltaic system, and so on [91-93]. The main objective of tracking control is to make the output of the plant track the output of a given reference model as close as possible [94]. As one of the typical control problems, tracking is far more difficult than the traditional stabilization especially for nonlinear systems. There are a great number of schemes to accomplish the tracking tasks, such as recursive technique, switching learning control, optimal adaptive control, error-constrained control, fuzzylogic-based control, sliding model control, and load-adaptive frequency control [95-98].

As an effective intelligent control method, sliding mode (or variable structure) control has recently attracted a lot of research interests from academy and industry because of its advantages in both robust properties and easy realization. Since it has adequate abilities to handle nonlinear problems and also adapt to the disturbances and parameter variations, the sliding mode control has been successively utilized to design the tracking controller. For example, a tracking problem for a class of nonlinear, time-varying systems has been solved in [99], where the state trajectory slides along a timevarying sliding surface in the state space. In [100], the sliding mode control method has been addressed for mobile robots with kinematics in two-dimensional polar coordinates, where the tracking errors in position and heading directions have been asymptotically stabilized by two designed controllers. 
For nonlinear NCSs design, many control schemes could be used to deal with the nonlinearities. These schemes include exact feedback linearization, sliding mode control, and adaptive control as examples, but these schemes have their own disadvantages; see, for example, [40]. Encouragingly, the T-S fuzzy model can be thought to be a universal approximator for nonlinear systems. In fact, T-S fuzzy model has proven to be a very good representation for a certain class of nonlinear NCSs. Therefore, the fuzzy-logic-based control plays an irreplaceable part in the tracking control of nonlinear NCSs. Some initial but inspiring results have appeared in the literature. For example, the cooperative fault tolerant fuzzy tracking control problem has been studied for the high order multiagent systems in [101], in which the tracking error of each follower node is ultimately bounded. Moreover, we can find that this design methodology requires relative state information between itself and its neighbors. For a class of nonlinear NCSs consisting of a nonlinear controlled plant, a tracked plant, sensors, a controller, and an actuator, the tracking control problem has been studied in [102] by using fuzzy control methods. In [103], a robust sampled-data output tracking controller has been investigated for a class of fuzzy networked systems.

\section{Fuzzy-Logic-Based Filtering and Fault Detection for Networked Systems}

Filtering and fault detection for networked systems are very significant issues in the control and signal processing fields, which have been comprehensively studied in the past years. As nonlinear intelligent control schemes, fuzzy-logic-based filtering and fault detection techniques have gained remarkable recognition due to their broad application insights and have been extensively applied to the networked systems. In this section, we will present some relevant literature of fuzzylogic-based filtering and fault detection for the networked systems and also make some discussions on the future development.

4.1. Fuzzy-Logic-Based Filtering. As is well known, the filtering problem is an active field of research in the past few decades because of the ever increasing demands for high quality signal, precise control, and higher performance of estimation. The main task of the filtering problems is to remove all kinds of noise signals from the measurements in order to estimate the unavailable information of a given system. For NCSs, there are a variety of reasons to address the filtering problem, and various methods have been proposed which include, but are not limited to, Kalman filtering, recursive filtering, robust filtering, optimal filtering, distributed filtering, tracking filtering, and gain-scheduled filtering methods; see, for example, [104-112].

As NCSs are actually a kind of distributed systems, they are affected by many uncertain factors, all of which will bring difficulties to the remote filter design. Fortunately, since the uncertain factors in NCSs can be effectively described in a stochastic setting, for instance, $H_{\infty}$ filtering problem has been studied for a class of uncertain nonlinear networked systems in [104], wherein the multiple stochastic time-varying communication delays and randomly occurring packet dropout are considered. One thing should be noted is that the obtained sufficient conditions are dependent on the occurrence probability of both the random communication delays and the missing measurements.

With the development of fuzzy linguistics, more and more researchers have started to employ the fuzzy logic theory to design the filters in the network environment over these years. Some practical and important results have been extensively reported for networked systems. For example, an admissible and robust state estimator has been investigated for a class of multichannel NCSs with multiple packet dropouts in [113], in which the T-S fuzzy-affine dynamic models with norm-bounded uncertainties have been introduced to describe the nonlinear plant, and a piecewise-quadratic Lyapunov function has been constructed to assist the analysis and design. In [114], the problem of $H_{\infty}$ filtering for a class of nonlinear time-varying delay systems with unideal communication links, the quantized measurements are assumed to be transmitted to the filter via networks. In [115], a full-order $H_{\infty}$ filter problem has been investigated for a class of NCSs with packet dropouts via T-S fuzzy model, where the transfer delays have been considered. In [113], a nonsynchronized-state estimation problem has been studied for the multichannel NCSs with multiple packet dropouts. In [74], a robust and reliable $H_{\infty}$ filtering problem has been investigated to ensure the mean-square stability of a class of nonlinear NCSs, where the T-S fuzzy model with its own uncertainties has been used to approximate the nonlinear dynamics of the plant. With the help of the fuzzy logic and Kalman filter theory, in [116], a global filter has been derived by fuzzy blending of the local filters designed for each subsystem. It is worth mentioning that the obtained global filter has been proved to be an unbiased state estimator.

4.2. Fuzzy-Logic-Based Fault Detection. Generally speaking, faults are unexpected deviations of system parameters from normal states, which extensively exist in controlled systems. Since the unpermitted deviation could prevent the controlled system from achieving prescribed performance requirements, the fault detection and isolation issues are of great significance in a real production environment. The basic idea of fault detection is to construct a residual signal and then determine a residual evaluation function so as to compare the predefined threshold with the constructed residual signal. Once the residual evaluation function is larger than the threshold, the alarm of fault should be indicated [117]. The diagram of the fuzzy-logic-based fault detection of NCSs is shown in Figure 2. In the past decades, research work on the fault detection has stirred consideration research attention; see, for example, [118-120].

On the other hand, although the networked systems offer various advantages in terms of enhanced resource utilization, reduced wiring, and reconfigurability, there are some disadvantages due to their complicated system structures such as multiple components, large scale, wide distribution, and high complexity. For this reason, the use of networks will unavoidably increase the fault occurrence rate in networked systems. Therefore, fault detection of networked systems 


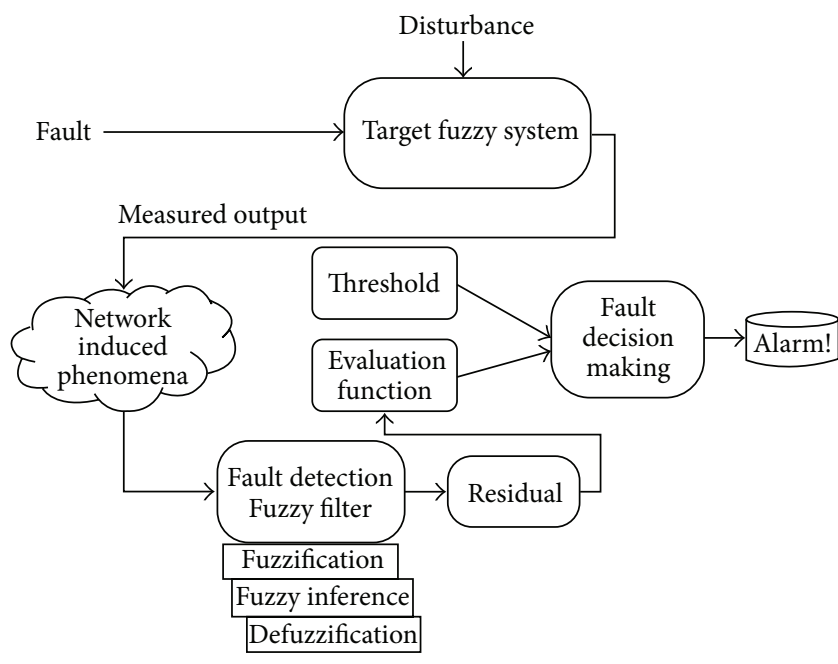

FIGURE 2: Fuzzy-logic-based fault detection of NCSs.

appears to be especially important and urgent, and it becomes an inevitable problem in NCSs. Over these years, fault detection for NCSs has attracted more and more attention; see, for example, [121-124].

Very recently, the fuzzy logic algorithms have been gradually introduced to accomplish the task of fault detection for NCSs, and some latest results have been published to reflect such a trend. For instance, a robust fault detector has been investigated for a class of nonlinear NCSs in [125], which is approximated by the uncertain T-S fuzzy model. The signal quantization has been considered in [126] when addressing the fuzzy-model-based fault detection for a class of nonlinear systems with networked measurements. Since the robust fault detection observer has been designed under network environment, the residual signal is sensitive to the fault but robust to exogenous disturbance. By using the switched system approach and some stochastic analytical skills, the fuzzy-model-based fault detection for a class of nonlinear systems with networked measurements has been studied in [126]. As a result, the estimation error between the residual and the fault is minimized by the designed fault detector. The fault detection problem for networked systems with global Lipschitz nonlinearities has been addressed in [127] with the help of fuzzy logic theory.

\section{Conclusion and Future Trends}

With the rapid progress of the computer science and network technology, the research on networked systems has achieved a rather mature status in the past years. On the other hand, the introduction of fuzzy logic theory provides an effective method for analysis and synthesis of networked systems, which also makes it possible to realize the intellectualization of NCSs. Moreover, as a nonlinear control scheme, the fuzzy control practices the reasoning process by language control rules, which can realize some nonlinear control laws for complicated NCSs. In this paper, we have summarized some recent advances on the fuzzy-logic-based control, filtering, and fault detection for the NCSs. Combination of the fuzzylogic-based control and some traditional algorithms in NCSs has been reviewed. Recent advances on the research of network-induced phenomena by using the fuzzy logic theory have been provided. Encouragingly, the application prospects of fuzzy logic theory in networked systems will be further broadened along with its development.

Some possible directions for future research are listed as follows.

(1) Two-Dimensional (2D) Networked Systems. Because of the potential applications in the gas absorption, the analysis of satellite weather photos, X-ray image enhancement, and so forth, $2 \mathrm{D}$ systems have received extensive interests in the past decades. Unfortunately, however, the research results on $2 \mathrm{D}$ networked systems have been scattered. As such, a practical problem to be solved is how to make full use of the fuzzy logic theory to accomplish the analysis and synthesis of 2D networked systems.

(2) Wireless Networked Control Systems (WNCSs). As is well known, the cabling and maintaining of wired NCSs are very time consuming and costing, which give rise to great inconvenience to the implementation of NCSs. Luckily, the WNCSs will be a promising solution due to their irreplaceable advantages, and thus the design of WNCSs will play an important role in industrial control in the future. Meanwhile, research on the new industrial wireless communication protocol suitable for WNCSs will be a promising topic.

(3) Quality-of-Service (QoS) of Networked Systems. As an inevitable issue, the QoS of networked systems is important to provide high quality communication to realize the real-time estimation and intelligent control. Unfortunately, however, the network-induced constraints will inevitably bring negative effects on QoS of NCSs. In this case, the design of a new communication mechanism from both hardware and software will be a significant task.

(4) Fuzzy Access Control in Open Networked Systems. Access control is a significant issue in an open network environment. In NCSs, secure communication and data access policy between sensors, actuators, and central controller will be a multifarious work. As an intelligent control method, the fuzzy logic theory will contribute a lot to the intellectualized design of access policy for NCSs.

(5) Smart Controller in Networked Systems. In some hash industry network, automatic control of production process is always susceptible to many factors, and, therefore, smart controller able to adapt to the changing environment of networks is much needed. Since the fuzzy-logic-based controller can incorporate the operator experience into the control systems, it will be expected to accomplish this goal.

In summary, high reliability, high intelligence, and quick adaptation of NCSs will be the main objectives in the future. For fuzzy logic theory, opportunities and challenges will coexist there. The research topics on fuzzy-logic-based 
control, filtering, and fault detection for networked systems would be attractive areas in the next decade.

\section{Conflict of Interests}

The authors declare that there is no conflict of interests regarding the publication of this paper.

\section{Acknowledgments}

This work was supported in part by the National Natural Science Foundation of China under Grants 61329301, 61374039,61473163 , and 61374127, the Hujiang Foundation of China under Grants C14002 and D15009, the Engineering and Physical Sciences Research Council (EPSRC) of the UK, the Royal Society of the UK, and the Alexander von Humboldt Foundation of Germany.

\section{References}

[1] S. Hu and Q. Zhu, "Stochastic optimal control and analysis of stability of networked control systems with long delay," Automatica, vol. 39, no. 11, pp. 1877-1884, 2003.

[2] G. C. Walsh and H. Ye, "Scheduling of networked control systems," IEEE Control Systems Magazine, vol. 21, no. 1, pp. 5765, 2001.

[3] W. Zhang, M. S. Branicky, and S. M. Phillips, "Stability of networked control systems," IEEE Control Systems Magazine, vol. 21, no. 1, pp. 84-97, 2001.

[4] J. P. Hespanha, P. Naghshtabrizi, and Y. Xu, "A survey of recent results in networked control systems," Proceedings of the IEEE, vol. 95, no. 1, pp. 138-162, 2007.

[5] Y. Tipsuwan and M.-Y. Chow, "Control methodologies in networked control systems," Control Engineering Practice, vol. 11, no. 10, pp. 1099-1111, 2003.

[6] G. C. Walsh, H. Ye, and L. G. Bushnell, "Stability analysis of networked control systems," IEEE Transactions on Control Systems Technology, vol. 10, no. 3, pp. 438-446, 2002.

[7] J. P. Hespanha, M. McLaughlin, G. S. Sukhatme, M. Akbarian, R. Garg, and W. Zhu, "Haptic collaboration over the internet," in Proceedings of the 5th PHANTOM Users Group Workshop, vol. 40, pp. 158-168, Los Angeles, Calif, USA, February 2001.

[8] P. Ogren, E. Fiorelli, and N. E. Leonard, "Cooperative control of mobile sensor networks: adaptive gradient climbing in a distributed environment," IEEE Transactions on Automatic Control, vol. 49, no. 8, pp. 1292-1302, 2004.

[9] P. Seiler and R. Sengupta, "An $H_{\infty}$ approach to networked control," IEEE Transactions on Automatic Control, vol. 50, no. 3, pp. 356-364, 2005.

[10] Z. Wang, F. Yang, D. W. C. Ho, and X. Liu, "Robust $H_{\infty}$ control for networked systems with random packet losses," IEEE Transactions on Systems, Man, and Cybernetics, Part B: Cybernetics, vol. 37, no. 4, pp. 916-924, 2007.

[11] S. Kolla, D. Border, and E. Mayer, "Fieldbus networks for control system implementations," in Proceedings of the Electrical Insulation Conference and Electrical Manufacturing \& Coil Winding Technology Conference, pp. 493-498, Indianapolis, Ind, USA, September 2003.

[12] D.-H. Choi and D.-S. Kim, "Wireless fieldbus for networked control systems using LR-WPAN," International Journal of Control, Automation and Systems, vol. 6, no. 1, pp. 119-125, 2008.
[13] M. Wu, H. Zhao, G.-P. Liu, and J.-H. She, "Networked control and supervision system based on LonWorks fieldbus and Intranet/Internet," Journal of Central South University of Technology, vol. 14, no. 2, pp. 260-265, 2007.

[14] N. Bibinagar and W.-J. Kim, "Switched ethernet-based realtime networked control system with multiple-client-server architecture," IEEE/ASME Transactions on Mechatronics, vol. 18, no. 1, pp. 104-112, 2013.

[15] P. L. Tang and C. W. de Silva, "Compensation for transmission delays in an ethernet-based control network using variablehorizon predictive control," IEEE Transactions on Control Systems Technology, vol. 14, no. 4, pp. 707-718, 2006.

[16] L. A. Zadeh, "Fuzzy sets," Information and Computation, vol. 8, no. 3, pp. 338-353, 1965.

[17] L. A. Zadeh, "Fuzzy algorithms," Information and Computation, vol. 12, pp. 94-102, 1968.

[18] L. A. Zadeh, "The concept of a linguistic variable and its application to approximate reasoning-I," Information Sciences, vol. 8, no. 3, pp. 199-249, 1975.

[19] G. Feng, "A survey on analysis and design of model-based fuzzy control systems," IEEE Transactions on Fuzzy Systems, vol. 14, no. 5, pp. 676-697, 2006.

[20] E. H. Mamdani, "Application of fuzzy algorithms for control of simple dynamic plant," Proceedings of the Institution of Electrical Engineers, vol. 121, no. 12, pp. 1585-1588, 1974.

[21] E. H. Mamdani and S. Assilian, "An experiment in linguistic synthesis with a fuzzy logic controller," International Journal of Man-Machine Studies, vol. 7, no. 1, pp. 1-13, 1975.

[22] M. Marseguerra and E. Zio, "Model-free fuzzy tracking control of a nuclear reactor," Annals of Nuclear Energy, vol. 30, no. 9, pp. 953-981, 2003.

[23] M. Marseguerra, E. Zio, and F. Cadini, "Genetic algorithm optimization of a model-free fuzzy control system," Annals of Nuclear Energy, vol. 32, no. 7, pp. 712-728, 2005.

[24] R.-E. Precup and H. Hellendoorn, "A survey on industrial applications of fuzzy control," Computers in Industry, vol. 62, no. 3, pp. 213-226, 2011.

[25] M. Sugeno, "On stability of fuzzy systems expressed by fuzzy rules with singleton consequents," IEEE Transactions on Fuzzy Systems, vol. 7, no. 2, pp. 201-224, 1999.

[26] Z. Bingiil, G. E. Cook, and A. M. Strauss, "Application of fuzzy logic to spatial thermal control in fusion welding," IEEE Transactions on Industry Applications, vol. 36, no. 6, pp. 15231530, 2000.

[27] O. Castillo, R. Martínez-Marroquín, P. Melin, F. Valdez, and J. Soria, "Comparative study of bio-inspired algorithms applied to the optimization of type- 1 and type- 2 fuzzy controllers for an autonomous mobile robot," Information Sciences, vol. 192, pp. 19-38, 2012.

[28] J.-I. Horiuchi and M. Kishimoto, "Application of fuzzy control to industrial bioprocesses in Japan," Fuzzy Sets and Systems, vol. 128, no. 1, pp. 117-124, 2002.

[29] S.-W. Liu, H.-P. Huang, C.-H. Lin, and I.-L. Chien, "A hybrid neural network model predictive control with zone penalty weights for type 1 diabetes mellitus," Industrial \& Engineering Chemistry Research, vol. 51, no. 26, pp. 9041-9060, 2012.

[30] M. Biglarbegian, W. W. Melek, and J. M. Mendel, "On the stability of interval type-2 tsk fuzzy logic control systems," IEEE Transactions on Systems, Man, and Cybernetics, Part B: Cybernetics, vol. 40, no. 3, pp. 798-818, 2010. 
[31] N. N. Karnik and J. M. Mendel, "Operations on type-2 fuzzy sets," Fuzzy Sets and Systems, vol. 122, no. 2, pp. 327-348, 2001.

[32] F. Lin and P. Chou, "Adaptive control of two-axis motion control system using interval type-2 fuzzy neural network," IEEE Transactions on Industrial Electronics, vol. 56, no. 1, pp. 178-193, 2009.

[33] Q. Liang and N. N. Karnik, "Connection admission control in ATM networks using survey-based type-2 fuzzy logic systems," IEEE Transactions on Systems, Man and Cybernetics Part C: Applications and Reviews, vol. 30, no. 3, pp. 329-339, 2000.

[34] J. M. Mendel, "Advances in type-2 fuzzy sets and systems," Information Sciences, vol. 177, no. 1, pp. 84-110, 2007.

[35] M. Maeda and S. Murakami, "An automobile tracking control with a fuzzy logic," in Proceedings of the 3rd Fuzzy Systems Symposium, pp. 61-66, 1987.

[36] G. Feng, Analysis and Synthesis of Fuzzy Control Systems: A Model-Based Approach, CRC Press, Boca Raton, Fla, USA, 2010.

[37] F.-H. Hsiao, C.-W. Chen, Y.-W. Liang, S.-D. Xu, and W.-L. Chiang, "T-S fuzzy controllers for nonlinear interconnected systems with multiple time delays," IEEE Transactions on Circuits and Systems I: Regular Papers, vol. 52, no. 9, pp. 1883-1893, 2005.

[38] H. Li, J. Yu, C. Hilton, and H. Liu, "Adaptive sliding-mode control for nonlinear active suspension vehicle systems using TS fuzzy approach," IEEE Transactions on Industrial Electronics, vol. 60, no. 8, pp. 3328-3338, 2013.

[39] B.-J. Rhee and S. Won, "A new fuzzy Lyapunov function approach for a Takagi-Sugeno fuzzy control system design," Fuzzy Sets and Systems, vol. 157, no. 9, pp. 1211-1228, 2006.

[40] C.-S. Tseng, B.-S. Chen, and H.-J. Uang, "Fuzzy tracking control design for nonlinear dynamic systems via T-S fuzzy model," IEEE Transactions on Fuzzy Systems, vol. 9, no. 3, pp. 381-392, 2001.

[41] B. Boukili, A. Hmamed, A. Benzaouia, and A. El Hajjaji, " $H_{\infty}$ filtering of two-dimensional T-S fuzzy systems," Circuits, Systems, and Signal Processing, vol. 33, no. 6, pp. 1737-1761, 2014.

[42] C.-W. Chen, "Neural network-based fuzzy logic parallel distributed compensation controller for structural system," Journal of Vibration and Control, vol. 19, no. 11, pp. 1709-1727, 2013.

[43] S. Hirche, T. Matiakis, and M. Buss, "A distributed controller approach for delay-independent stability of networked control systems," Automatica, vol. 45, no. 8, pp. 1828-1836, 2009.

[44] H. Yan, X. Huang, M. Wang, and H. Zhang, "Delay-dependent stability criteria for a class of networked control systems with multi-input and multi-output," Chaos, Solitons \& Fractals, vol. 34, no. 3, pp. 997-1005, 2007.

[45] H. Gao, T. Chen, and J. Lam, "A new delay system approach to network-based control," Automatica, vol. 44, no. 1, pp. 39-52, 2008.

[46] L. Zhang, Y. Shi, T. Chen, and B. Huang, "A new method for stabilization of networked control systems with random delays," IEEE Transactions on Automatic Control, vol. 50, no. 8, pp. 11771181, 2005.

[47] W.-Y. Chiu, B.-S. Chen, and H. V. Poor, "A multiobjective approach for source estimation in fuzzy networked systems," IEEE Transactions on Circuits and Systems. I. Regular Papers, vol. 60, no. 7, pp. 1890-1900, 2013.

[48] M. Cardei and J. Wu, "Energy-efficient coverage problems in wireless ad-hoc sensor networks," Computer Communications, vol. 29, no. 4, pp. 413-420, 2006.

[49] J.-J. Xiao, S. Cui, Z.-Q. Luo, and A. J. Goldsmith, "Power scheduling of universal decentralized estimation in sensor networks," IEEE Transactions on Signal Processing, vol. 54, no. 2, pp. 413-422, 2006.

[50] M. S. Mahmoud and A.-W. A. Saif, "Robust quantized approach to fuzzy networked control systems," IEEE Journal on Emerging and Selected Topics in Circuits and Systems, vol. 2, no. 1, pp. 7181, 2012.

[51] C. Peng and Y.-C. Tian, "Networked $H_{\infty}$ control of linear systems with state quantization," Information Sciences, vol. 177, no. 24, pp. 5763-5774, 2007.

[52] R. Yang, P. Shi, G.-P. Liu, and H. Gao, "Network-based feedback control for systems with mixed delays based on quantization and dropout compensation," Automatica, vol. 47, no. 12, pp. 2805-2809, 2011.

[53] Z. Gao, T. Breikin, and H. Wang, "Reliable observer-based control against sensor failures for systems with time delays in both state and input," IEEE Transactions on Systems, Man, and Cybernetics, Part A: Systems and Humans, vol. 38, no. 5, pp. 1018-1029, 2008.

[54] S. R. Naidu, E. Zafiriou, and T. J. McAvoy, "Use of neural networks for sensor failure detection in a control system," IEEE Control Systems Magazine, vol. 10, no. 3, pp. 49-55, 1990.

[55] L. Qin, X. He, and D. Zhou, "A survey of fault diagnosis for swarm systems," Systems Science \& Control Engineering, vol. 2, no. 1, pp. 13-23, 2014.

[56] D. Ding, Z. Wang, J. Lam, and B. Shen, "Finite-horizon $H_{\infty}$ control for discrete time-varying systems with randomly occurring nonlinearities and fading measurements," IEEE Transactions on Automatic Control, 2015.

[57] D. Ding, Z. Wang, B. Shen, and H. Dong, "Envelope-constrained $H_{\infty}$ filtering with fading measurements and randomly occurring nonlinearities: The finite horizon case," Automatica, vol. 55, pp. 37-45, 2015.

[58] S. Zhang, Z. Wang, D. Ding, and H. Shu, " $H_{\infty}$ fuzzy control with randomly occurring infinite distributed delays and channel fadings," IEEE Transactions on Fuzzy Systems, vol. 22, no. 1, pp. 189-200, 2014.

[59] W. Zeng and M.-Y. Chow, "Optimal tradeoff between performance and security in networked control systems based on coevolutionary algorithms," IEEE Transactions on Industrial Electronics, vol. 59, no. 7, pp. 3016-3025, 2012.

[60] S. Liu, P. X. Liu, and A. El Saddik, "A stochastic game approach to the security issue of networked control systems under jamming attacks," Journal of the Franklin Institute. Engineering and Applied Mathematics, vol. 351, no. 9, pp. 4570-4583, 2014.

[61] S. Amin, G. A. Schwartz, and S. S. Sastry, "Security of interdependent and identical networked control systems," Automatica, vol. 49, no. 1, pp. 186-192, 2013.

[62] Q. Liu, Z. Wang, X. He, and D. Zhou, "A survey of eventbased strategies on control and estimation," Systems Science and Control Engineering, vol. 2, no. 1, pp. 90-97, 2014.

[63] C. Lin, Z. Wang, and F. Yang, "Observer-based networked control for continuous-time systems with random sensor delays," Automatica, vol. 45, no. 2, pp. 578-584, 2009.

[64] D. Yue, Q.-L. Han, and C. Peng, "State feedback controller design of networked control systems," IEEE Transactions on Circuits and Systems II: Express Briefs, vol. 51, no. 11, pp. 640644, 2004.

[65] J. Qiu, G. Feng, and H. Gao, "Fuzzy-model-based piecewise $H_{\infty}$ static-output-feedback controller design for networked nonlinear systems," IEEE Transactions on Fuzzy Systems, vol. 18, no. 5, pp. 919-934, 2010. 
[66] X. Zhang, G. Lu, and Y. Zheng, "Stabilization of networked stochastic time-delay fuzzy systems with data dropout," IEEE Transactions on Fuzzy Systems, vol. 16, no. 3, pp. 798-807, 2008.

[67] C. Peng and T. C. Yang, "Communication-delay-distributiondependent networked control for a class of T-S fuzzy systems," IEEE Transactions on Fuzzy Systems, vol. 18, no. 2, pp. 326-335, 2010.

[68] H. Zhang, M. Li, J. Yang, and D. Yang, "Fuzzy model-based robust networked control for a class of nonlinear systems," IEEE Transactions on Systems, Man, and Cybernetics, Part A: Systems and Humans, vol. 39, no. 2, pp. 437-447, 2009.

[69] S. H. Kim, "T-S fuzzy control design for a class of nonlinear networked control systems," Nonlinear Dynamics, vol. 73, no. 12, pp. 17-27, 2013.

[70] S. Chae, S. K. Nguang, and W. Wang, "Robust $H_{\infty}$ fuzzy control of discrete nonlinear networked control systems: a SOS approach," Journal of the Franklin Institute. Engineering and Applied Mathematics, vol. 351, no. 8, pp. 4065-4083, 2014.

[71] J. Qiu, G. Feng, and H. Gao, "Asynchronous output-feedback control of networked nonlinear systems with multiple packet dropouts: T-S fuzzy affine model-based approach," IEEE Transactions on Fuzzy Systems, vol. 19, no. 6, pp. 1014-1030, 2011.

[72] K. C. Lee, S. Lee, and M. H. Lee, "Remote fuzzy logic control of networked control system via profibus-DP," IEEE Transactions on Industrial Electronics, vol. 50, no. 4, pp. 784-792, 2003.

[73] C. Peng, M.-R. Fei, and E. Tian, "Networked control for a class of T-S fuzzy systems with stochastic sensor faults," Fuzzy Sets and Systems, vol. 212, pp. 62-77, 2013.

[74] E. Tian and D. Yue, "Reliable $H_{\infty}$ filter design for T-S fuzzy model-based networked control systems with random sensor failure," International Journal of Robust and Nonlinear Control, vol. 23, no. 1, pp. 15-32, 2013.

[75] H. Zhang, J. Yang, and C.-Y. Su, "T-S fuzzy-model-based robust $H_{\infty}$ design for networked control systems with uncertainties," IEEE Transactions on Industrial Informatics, vol. 3, no. 4, pp. 289-301, 2007.

[76] H. Zhang, D. Yang, and T. Chai, "Guaranteed cost networked control for T-S fuzzy systems with time delays," IEEE Transactions on Systems, Man and Cybernetics Part C: Applications and Reviews, vol. 37, no. 2, pp. 160-172, 2007.

[77] L. Cai, Z. Yang, J. Yu, and Z. Zhang, "Switching fuzzy guaranteed cost control for nonlinear networked control systems," Mathematical Problems in Engineering, vol. 2014, Article ID 539617, 12 pages, 2014.

[78] A. Fadaei and K. Salahshoor, "Improving the control performance of networked control systems using a new fuzzy PID," in Proceedings of the IEEE International Symposium on Industrial Electronics (ISIE '08), pp. 2066-2071, Cambridge, UK, June-July 2008.

[79] K. J. Åström and T. Hägglund, "The future of PID control," Control Engineering Practice, vol. 9, no. 11, pp. 1163-1175, 2001.

[80] J. H. Kim and S. J. Oh, "A fuzzy PID controller for nonlinear and uncertain systems," Soft Computing, vol. 4, no. 2, pp. 123129, 2000.

[81] M. A. Rodrigo, A. Seco, J. Ferrer, J. M. Penya-Roja, and J. L. Valverde, "Nonlinear control of an activated sludge aeration process: use of fuzzy techniques for tuning PID controllers," ISA Transactions, vol. 38, no. 3, pp. 231-241, 1999.

[82] A. Fadaei and K. Salahshoor, "Design and implementation of a new fuzzy PID controller for networked control systems," ISA Transactions, vol. 47, no. 4, pp. 351-361, 2008.
[83] I. Pan, S. Das, and A. Gupta, "Tuning of an optimal fuzzy PID controller with stochastic algorithms for networked control systems with random time delay," ISA Transactions, vol. 50, no. 1, pp. 28-36, 2011.

[84] Z.-M. Zou, X.-Y. Liu, and J.-H. Zeng, "Networked control systems based on new Smith predictor and fuzzy-PID controller," Applied Mechanics and Materials, vol. 530-531, pp. 999-1002, 2014.

[85] K. J. Åström, "Theory and applications of adaptive control: a survey," Automatica, vol. 19, no. 5, pp. 471-486, 1983.

[86] B. Yao and M. Tomizuka, "Adaptive robust control of SISO nonlinear systems in a semi-strict feedback form," Automatica, vol. 33, no. 5, pp. 893-900, 1997.

[87] A. O. Esogbue and J. A. Murrell, "Advances in fuzzy adaptive control," Computers \& Mathematics with Applications, vol. 27, no. 9-10, pp. 29-35, 1994.

[88] Y. Yang, C. Hua, and X. Guan, "Adaptive fuzzy finite-time coordination control for networked nonlinear bilateral teleoperation system," IEEE Transactions on Fuzzy Systems, vol. 22, no. 3, pp. 631-641, 2014.

[89] H. Yu, C. Shao, and D. Zhou, "Adaptive single neuron PID control with fuzzy and self-tuning in networked control systems," Applied Mechanics and Materials, vol. 235, pp. 152-157, 2012.

[90] P. Hajebi and S. M. T. AlModarresi, "Online adaptive Smith predictor with fuzzy controller for networked control systems," in Proceedings of the 2nd International Conference on Control, Instrumentation and Automation (ICCIA '11), pp. 594-599, Shiraz, Iran, December 2011.

[91] T.-C. Lee, K.-T. Song, C.-H. Lee, and C.-C. Teng, "Tracking control of unicycle-modeled mobile robots using a saturation feedback controller," IEEE Transactions on Control Systems Technology, vol. 9, no. 2, pp. 305-318, 2001.

[92] N. Patcharaprakiti, S. Premrudeepreechacharn, and Y. Sriuthaisiriwong, "Maximum power point tracking using adaptive fuzzy logic control for grid-connected photovoltaic system," Renewable Energy, vol. 30, no. 11, pp. 1771-1788, 2005.

[93] M. Sampei, T. Tamura, T. Kobayashi, and N. Shibui, "Arbitrary path tracking control of articulated vehicles using nonlinear control theory," IEEE Transactions on Control Systems Technology, vol. 3, no. 1, pp. 125-131, 1995.

[94] H. Gao and T. Chen, "Network-based output tracking control," IEEE Transactions on Automatic Control, vol. 53, no. 3, pp. 655$667,2008$.

[95] S.-I. Furuya, T. Maruhashi, Y. Izuno, and M. Nakaoka, "Loadadaptive frequency tracking control implementation of twophase resonant inverter for ultrasonic motor," IEEE Transactions on Power Electronics, vol. 7, no. 3, pp. 542-550, 1992.

[96] Z.-P. Jiang and H. Nijmeijer, "A recursive technique for tracking control of nonholonomic systems in chained form," IEEE Transactions on Automatic Control, vol. 44, no. 2, pp. 265-279, 1999.

[97] W. Luo, Y.-C. Chu, and K.-V. Ling, "Inverse optimal adaptive control for attitude tracking of spacecraft," IEEE Transactions on Automatic Control, vol. 50, no. 11, pp. 1639-1654, 2005.

[98] P. R. Ouyang, W. J. Zhang, and M. M. Gupta, "An adaptive switching learning control method for trajectory tracking of robot manipulators," Mechatronics, vol. 16, no. 1, pp. 51-61, 2006.

[99] J.-J. Slotine and S. S. Sastry, "Tracking control of non-linear systems using sliding surfaces, with application to robot manipulators," International Journal of Control, vol. 38, no. 2, pp. 465492, 1983. 
[100] D. Chwa, "Sliding-mode tracking control of nonholonomic wheeled mobile robots in polar coordinates," IEEE Transactions on Control Systems Technology, vol. 12, no. 4, pp. 637-644, 2004.

[101] Q. Shen, B. Jiang, P. Shi, and J. Zhao, "Cooperative adaptive fuzzy tracking control for networked unknown nonlinear multi-agent systems with time-varying actuator faults," IEEE Transactions on Fuzzy Systems, vol. 22, no. 3, pp. 494-504, 2014.

[102] X. Jia, D. Zhang, X. Hao, and N. Zheng, "Fuzzy $H_{\infty}$ tracking control for nonlinear networked control systems in T-S fuzzy model," IEEE Transactions on Systems, Man, and Cybernetics, Part B: Cybernetics, vol. 39, no. 4, pp. 1073-1079, 2009.

[103] S. Wen, Z. Zeng, and T. Huang, "Robust $H_{\infty}$ output tracking control for fuzzy networked systems with stochastic sampling and multiplicative noise," Nonlinear Dynamics, vol. 70, no. 2, pp. 1061-1077, 2012.

[104] H. Dong, Z. Wang, and H. Gao, "Robust $H_{\infty}$ filtering for a class of nonlinear networked systems with multiple stochastic communication delays and packet dropouts," IEEE Transactions on Signal Processing, vol. 58, no. 4, pp. 1957-1966, 2010.

[105] J. Hu, Z. Wang, B. Shen, and H. Gao, "Quantised recursive filtering for a class of nonlinear systems with multiplicative noises and missing measurements," International Journal of Control, vol. 86, no. 4, pp. 650-663, 2013.

[106] J. Liang, F. Sun, and X. Liu, "Finite-horizon $H_{\infty}$ filtering for time-varying delay systems with randomly varying nonlinearities and sensor saturations," Systems Science \& Control Engineering, vol. 2, no. 1, pp. 108-118, 2014.

[107] H. Ramoser, J. Müller-Gerking, and G. Pfurtscheller, “Optimal spatial filtering of single trial EEG during imagined hand movement," IEEE Transactions on Rehabilitation Engineering, vol. 8, no. 4, pp. 441-446, 2000.

[108] R. A. Singer, "Estimating optimal tracking filter performance for manned maneuvering targets," IEEE Transactions on Aerospace and Electronic Systems, vol. 6, no. 4, pp. 473-483, 1970.

[109] Y. Shi, H. Fang, and M. Yan, "Kalman filter-based adaptive control for networked systems with unknown parameters and randomly missing outputs," International Journal of Robust and Nonlinear Control, vol. 19, no. 18, pp. 1976-1992, 2009.

[110] B. Shen, Z. Wang, H. Shu, and G. Wei, "Robust $H_{\infty}$ finitehorizon filtering with randomly occurred nonlinearities and quantization effects," Automatica, vol. 46, no. 11, pp. 1743-1751, 2010.

[111] Z. Wang, X. Liu, Y. Liu, J. Liang, and V. Vinciotti, "An extended kalman filtering approach to modeling nonlinear dynamic gene regulatory networks via short gene expression time series," IEEE/ACM Transactions on Computational Biology and Bioinformatics, vol. 6, no. 3, pp. 410-419, 2009.

[112] G. Wei, Z. Wang, B. Shen, and M. Li, "Probability-dependent gain-scheduled filtering for stochastic systems with missing measurements," IEEE Transactions on Circuits and Systems II: Express Briefs, vol. 58, no. 11, pp. 753-757, 2011.

[113] J. Qiu, G. Feng, and H. Gao, "Nonsynchronized-state estimation of multichannel networked nonlinear systems with multiple packet dropouts via T-S fuzzy-affine dynamic models," IEEE Transactions on Fuzzy Systems, vol. 19, no. 1, pp. 75-90, 2011.

[114] R. Lu, H. Wu, and J. Bai, "Networked $H_{\infty}$ filtering for T-S fuzzy systems with quantization and data dropouts," Journal of the Franklin Institute. Engineering and Applied Mathematics, vol. 351, no. 6, pp. 3126-3144, 2014.

[115] B. Jiang, Z. Mao, and P. Shi, " $H_{\infty}$-filter design for a class of networked control systems via T-S fuzzy-model approach,"
IEEE Transactions on Fuzzy Systems, vol. 18, no. 1, pp. 201-208, 2010.

[116] F. Yang and H. Fang, "State estimation for networked control systems based on fuzzy Kalman filter," Information and Control, vol. 36, no. 3, pp. 257-260, 2007.

[117] L. Wu and D. W. C. Ho, "Fuzzy filter design for Itô stochastic systems with application to sensor fault detection," IEEE Transactions on Fuzzy Systems, vol. 17, no. 1, pp. 233-242, 2009.

[118] C. Edwards, S. K. Spurgeon, and R. J. Patton, "Sliding mode observers for fault detection and isolation," Automatica, vol. 36, no. 4, pp. 541-553, 2000.

[119] V. Venkatasubramanian, R. Rengaswamy, and S. N. Kavuri, "A review of process fault detection and diagnosis: part II: qualitative models and search strategies," Computers \& Chemical Engineering, vol. 27, no. 3, pp. 313-326, 2003.

[120] B. M. Wise and N. B. Gallagher, "The process chemometrics approach to process monitoring and fault detection," Journal of Process Control, vol. 6, no. 6, pp. 329-348, 1996.

[121] X. He, Z. Wang, and D. H. Zhou, "Robust fault detection for networked systems with communication delay and data missing," Automatica, vol. 45, no. 11, pp. 2634-2639, 2009.

[122] Z. Mao, B. Jiang, and P. Shi, "Protocol and fault detection design for nonlinear networked control systems," IEEE Transactions on Circuits and Systems II: Express Briefs, vol. 56, no. 3, pp. 255-259, 2009.

[123] C. Peng, D. Yue, E. Tian, and Z. Gu, "Observer-based fault detection for networked control systems with network quality of services," Applied Mathematical Modelling, vol. 34, no. 6, pp. 1653-1661, 2010.

[124] Y. Wang, S. X. Ding, H. Ye, and G. Wang, "A new fault detection scheme for networked control systems subject to uncertain time-varying delay," IEEE Transactions on Signal Processing, vol. 56, no. 10, part 2, pp. 5258-5268, 2008.

[125] H. Huang, D. Xie, and X. Han, "Fault Detection for a class of T-S fuzzy-model-based nonlinear networked control system," Information and Control, vol. 38, no. 6, pp. 703-710, 2009.

[126] D. Zhang, Q.-G. Wang, L. Yu, and H. Song, "Fuzzy-model-based fault detection for a class of nonlinear systems with networked measurements," IEEE Transactions on Instrumentation and Measurement, vol. 62, no. 12, pp. 3148-3159, 2013.

[127] X. Wan and H. Fang, "Fault detection for networked nonlinear systems with time delays and packet dropouts," Circuits, Systems, and Signal Processing, vol. 31, no. 1, pp. 329-345, 2012. 


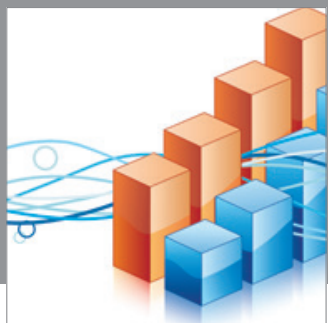

Advances in

Operations Research

mansans

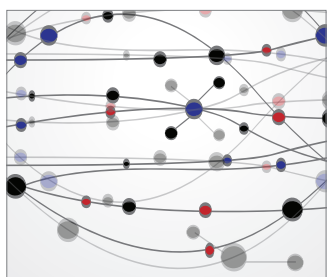

The Scientific World Journal
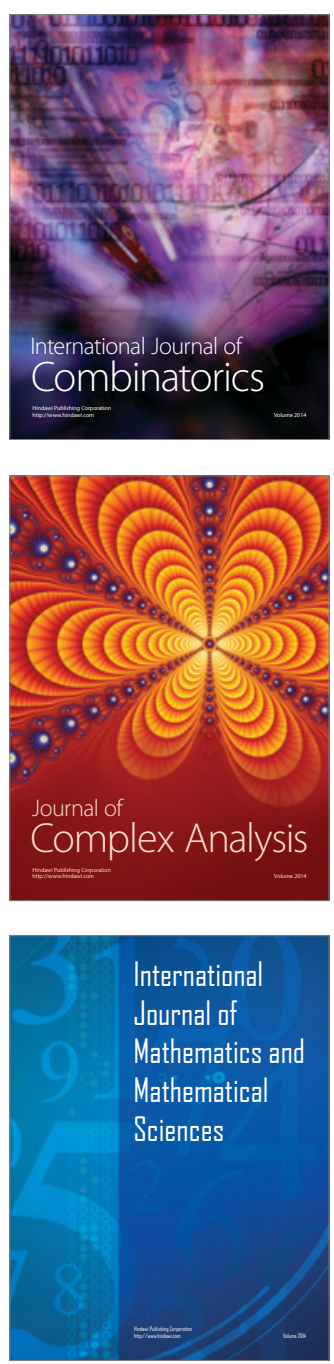
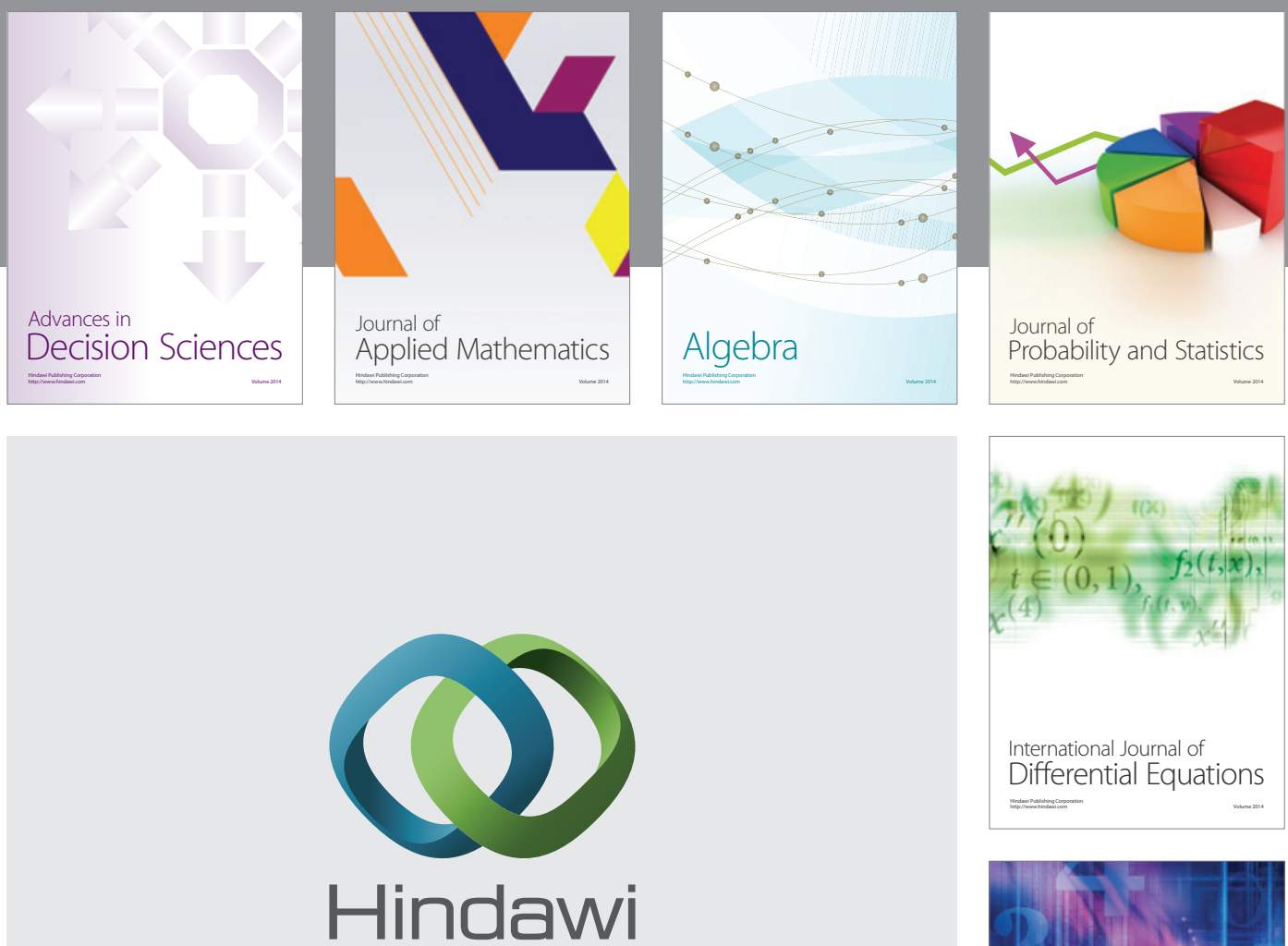

Submit your manuscripts at http://www.hindawi.com
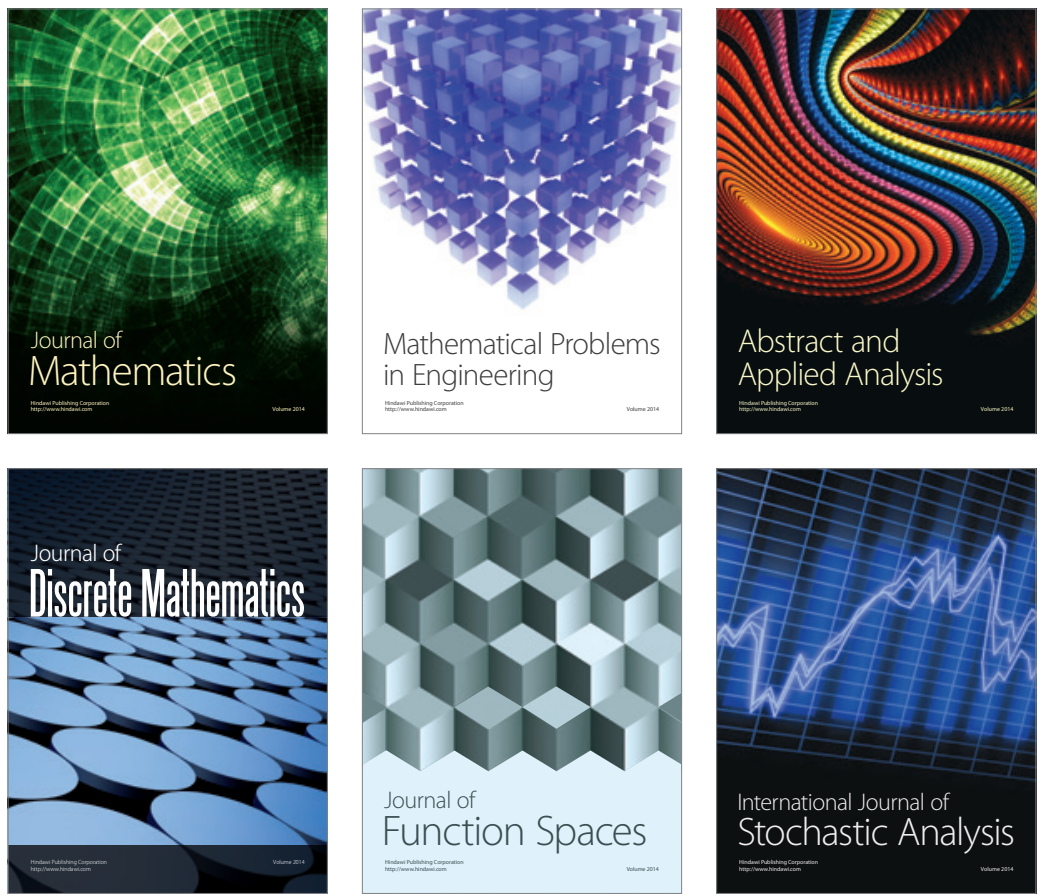

Journal of

Function Spaces

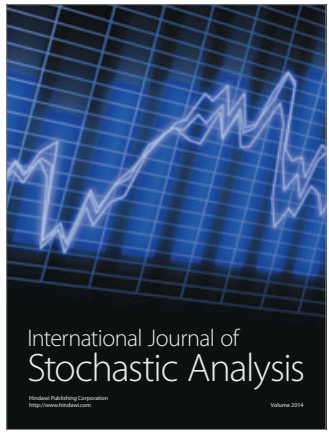

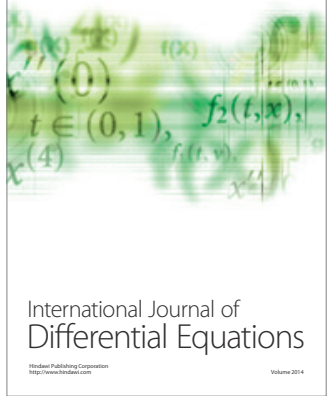
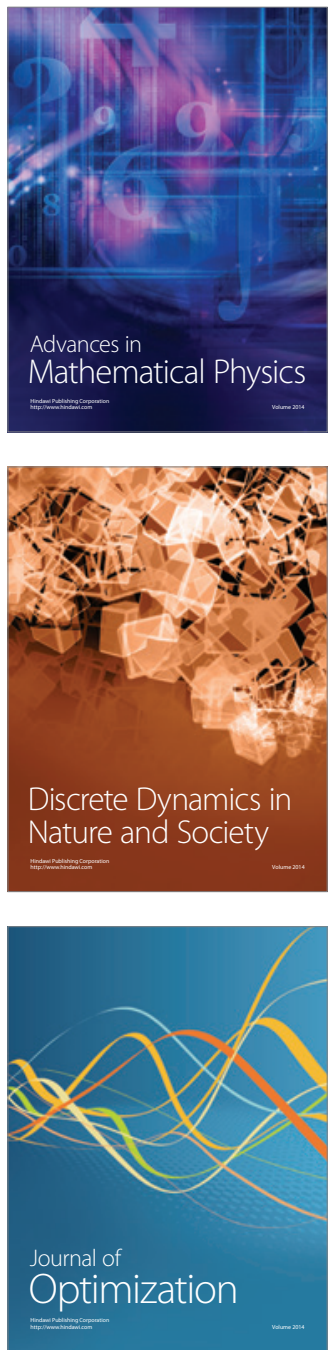